\title{
Feasibility of a Nuclear Fission Reactor at the Center of the Earth as the Energy Source for the Geomagnetic Field
}

\author{
J. Marvin HERNDON \\ Herndon Science \& Software, Inc., 11044 Red Rock Drive, San Diego, CA 92131, U.S.A. \\ (Received February 28, 1992; Revised January 22, 1993)
}

\begin{abstract}
Ideas have previously been advanced suggesting the possibility that uranium exists within the Earth's core. In the present paper, evidence is presented for the existence within the Earth's core of substantial quantities of uranium and thorium. The concept of an accumulation of uranium in the core of the Earth functioning as a nuclear fission breeder reactor is presented. Means for concentrating actinide elements within the Earth's core and for separating actinide elements from reactor poisons are disclosed. Nuclear reactor feasibility is demonstrated by Fermi's $k_{\infty}$ in excess of unity for times in the geological past. The concept that the Earth's geomagnetic dynamo is driven by nuclear fission energy is discussed as is the concept that the frequent, but irregular, polarity reversals of the geomagnetic field have their origins in intermittent nuclear reactor output. Although great uncertainty exists in estimates of the abundances of the actinide elements in the core of the Earth and in details of the chemistry of the core, the results of the present paper indicate if uranium and thorium exist in the core of the Earth as elements or compounds, as evidence indicates, the actinides: (1) would be the most dense matter in the Earth; (2) would tend to concentrate at the center of the Earth; (3) would tend to be separated on the basis of density from less dense reactor poisons; and (4) if accumulated 3000 million years ago, would be able to initiate self-sustaining nuclear fission chain reactions which may continue to the present through fuel breeding reactions. The magnitude of available nuclear fission energy release throughout geological time is of major geophysical importance and is more than sufficient to power the geomagnetic field.
\end{abstract}

\section{Introduction}

In 1919, Larmor suggested that the Sun's magnetic field might be sustained by a mechanism similar to a self-exciting dynamo (LARMOR, 1919). Almost a half century ago, that concept was extended with the suggestion (ELSASSER, 1939, 1946; BULLARD, 1949) that fluid motions in the electrically conducting core of the Earth might cause the core to act like a self-exciting dynamo, producing electric currents to sustain the geomagnetic field (see, for example, RIKITAKE, 1966). Various ideas have been discussed as energy sources for the geomagnetic field, including thermal convection in the outer core caused by the decay energy from naturally occurring radioactivities $\left(\mathrm{U}, \mathrm{Th},{ }^{40} \mathrm{~K}\right)$ and compositionally driven convection caused by the presumed growth of a dense inner core from a progressively less dense fluid core (ELSASSER, 1939, 1950; GUBBINS and MASTERS, 1979; VERHOOGEN, 1980; LOPER and ROBERTS, 1983). ELSASSER (1950) suggested the radioactive decay energy of uranium and thorium concentrated at the center of the Earth as causing thermal convection in the fluid core. Later, FERBER et al. (1984) again suggested the radioactive decay energy of uranium and supported that suggestion with experimental data demonstrating the tendency of uranium to alloy with iron at high temperatures. The present paper presents evidence for the existence of uranium and thorium in the Earth's core based upon the identification of the interior parts of the Earth with corresponding parts of the Abee enstatite chondrite meteorite. The feasibility of uranium concentrating in the Earth's core and undergoing self-sustaining nuclear fission chain reactions is demonstrated. 
2. Relationship between the Components of the Interior of the Earth and the Components of the Abee Enstatite Chondrite Meteorite

The physical states, divisions, and densities of the interior parts of the Earth are deduced from the travel times of earthquake waves, from the moment of inertia of the Earth, and from the fundamental periods of free oscillations of the whole Earth (DZIEWONSKI and GILBERT, 1972; DZIEWONSKI and ANDERSON, 1981). Chemical compositions of the interior portions of the Earth, on the other hand, are inferred from observations of meteorites (HERNDON, 1980, 1982).

Most chondritic meteorites, with the exception of some of the more oxidized carbonaceous chondrites, contain iron metal and iron sulfide as well as silicate minerals and oxides. If melted in a gravitational field, iron metal and the constituents that dissolve in molten iron, for example, sulfides, will form a dense, immiscible, liquid "alloy" that settles beneath the silicate-oxide "slag" in a manner analogous to steel on a steel hearth. Upon subsequent cooling, the alloy and slag components may further differentiate. The Earth is analogous to a spherical steel hearth, the core corresponding to the alloy and the mantle corresponding to the slag.

Only the three elements, magnesium, silicon, and iron, together with their associated oxygen and sulfur, comprise at least ninety percent of the mass of chondritic meteorites. Figure 1 shows the mass ratio of alloy to silicate-oxide slag, calculated from published data on 147 meteorites, expressed as a function of the mean atomic number of the alloy. Similar parameters for the Earth, deduced from seismological investigations, are also shown. Many of the data points of the 131 ordinary (H, L, LL group) chondrites and the 6 metal-bearing carbonaceous chondrites overlap. The data points of the 10 highly reduced enstatite chondrites are quite distinct from the other chondrites and are strikingly similar to the Earth's core to mantle ratio shown in Fig. 1.

Nickel silicide, $\mathrm{Ni}_{2} \mathrm{Si}$, an important precipitate from a molten enstatite chondrite alloy, occurs as a lamellar exsolution from silicon-bearing iron metal (WAI, 1970; WASSON and WAI, 1970) as well as a more massive form intimately associated with iron metal and iron sulfide in some enstatite chondrites

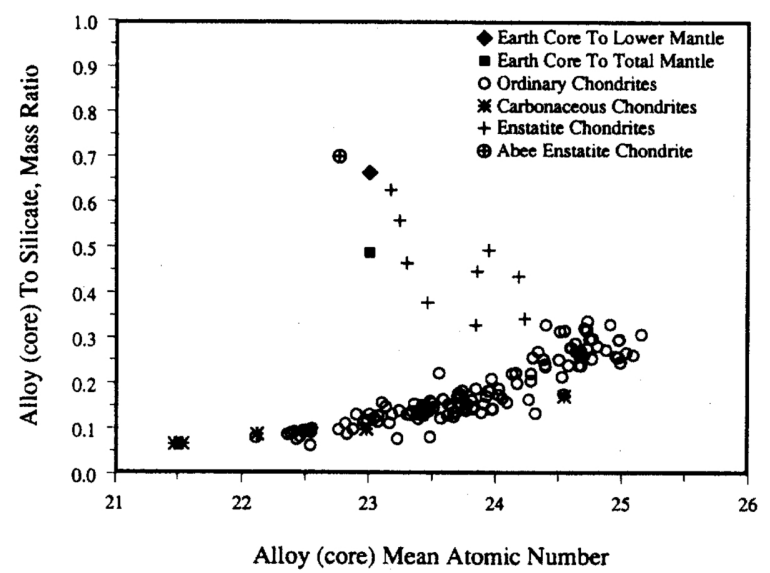

Fig. 1. The alloy to slag mass ratio of 147 meteorites as a function of their alloy mean atomic number. The Earth's core to lower mantle mass ratio, plotted against the mean atomic number of the core, is almost identical to the Abee enstatite chondrite in this figure. Even the whole-Earth alloy to slag mass ratio is similar to ten enstatite chondrites and is unlike the 137 other chondrites shown. Meteorite data from KeIL (1962, 1968), WIIK (1969), BAEDECKER and WASSON (1975), JAROSEWICH and DodD (1985), KALLEMEYN et al. (1989); Earth data from KNOPOFF and MACDONALD (1960) and DZIEWONSKI and ANDERSON (1981). 
(REED, 1968; RAMDOHR, 1973). Figure 2, from data for the same 147 chondrites plotted in Fig. 1, shows theoretical maximum nickel silicide content, based on the limitations imposed by the respective amounts of silicon and nickel present in the alloy. Expressed as a function of mean atomic mass of the alloy, the individual results are plotted as a ratio of theoretical nickel silicide to alloy mass in Fig. 2 together with the mass ratio of the Earth's inner core to total core obtained from seismological investigations. Of the various chondrites, only the silicon-bearing alloys of enstatite chondrites are capable of precipitating nickel silicide; the 137 data points representing the non-silicon-bearing alloys of the more oxidized ordinary (H, L, LL group) chondrites and carbonaceous chondrites have no nickel silicide and are therefore not plotted in Fig. 2.

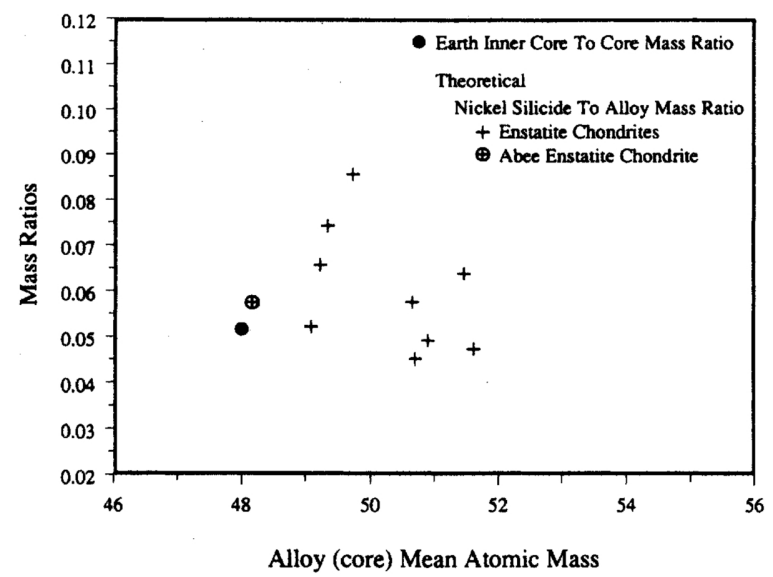

Fig. 2. The theoretical nickel silicide to alloy mass ratio of 10 enstatite chondrite meteorites as a function of their alloy mean atomic mass. The Earth's inner core to core mass ratio, plotted against the mean atomic mass of the core, is almost identical to the Abee enstatite chondrite in this figure, is similar other enstatite chondrites, and is unlike the 137 other more oxidized chondrites which are devoid of nickel silicide and, therefore, are not plotted. Meteorite data from KEIL (1968) and BAEDECKER and WASSON (1975); Earth data from KNOPOFF and MACDONALD (1960) and DzIEwONSKI and ANDERSON (1981).

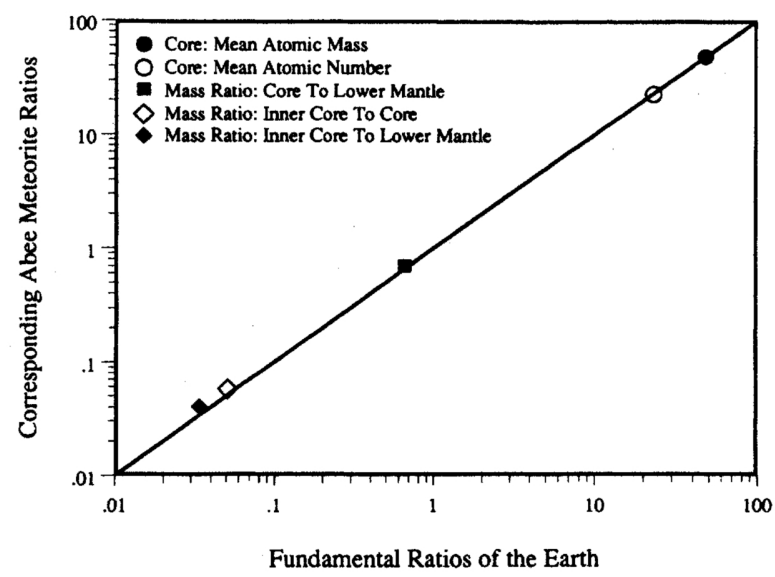

Fig. 3. Comparison of fundamental mass ratios of the Earth with corresponding mass ratios of the alloy and slag components of the Abee enstatite chondrite. To facilitate comparison, a line of perfect correlation is shown. The core and lower mantle of the Earth, by these fundamental quantitative relationships, are virtually identical to the alloy and slag of the Abee meteorite. 
The data shown in Figs. 1 and 2 identify the lower mantle and core of the Earth with the slag and alloy components of the Abee enstatite chondrite and support the suggestion by the author that the inner core of the Earth is composed of nickel silicide (HERNDON, 1979), rather than partially crystallized nickel-iron metal, as previously assumed (BULLEN, 1946). Figure 3 relates seismologically determined fundamental ratios of the Earth with mineralogically determined corresponding ratios of the Abee meteorite, as abstracted from the data presented in Figs. 1 and 2.

Identification of the interior portions of the Earth, the lower mantle and core, with the slag and alloy components of the Abee enstatite chondrite (Fig. 3) is important in providing a basis for understanding the compositions of the interior eighty-two percent of the Earth. This identification allows a reasonable estimation of the compositions of the lower mantle and core of the Earth to be made by analyzing the slag and alloy of the Abee meteorite. Although pressures that exist within the Earth can in principle disproportionate complex oxides into metal and simple oxides, such disproportionation is not expected for the slag of the Abee meteorite because (1) the Abee silicates are essentially combinations of simple oxides, (2) the Abee slag contains extremely little $\mathrm{FeO}$, and (3) the major component of the Abee slag, $\mathrm{MgSiO}_{3}$, is known from experiment to be stable at lower mantle pressures (LIU, 1976; ITO and MATSUI, 1978).

\section{Chemical Composition of the Core and Lower Mantle of the Earth}

Only nine chemical elements account for approximately ninety-eight percent of the mass of the Abee meteorite. The relative proportions of these nine elements, as partitioned between the alloy and slag components of the Abee meteorite and, by inference, between the total core and lower mantle of the Earth are shown in Fig. 4, a plot of elemental mass ratios, $\mathrm{E}_{i} / \mathrm{Fe}$; estimates of the absolute amounts of those nine elements in the Earth's core and lower mantle are set forth in Table 1.

In the Abee meteorite, as shown in Fig. 4, essentially all of the iron, nickel and sulfur occur in the alloy portion as a consequence of the extremely limited available oxygen. Moreover, certain normally lithophile elements (for example, magnesium and calcium) in part also occur as sulfide compounds that, at elevated temperatures and pressures, would, with iron, nickel, and sulfur, form a dense liquid solution,

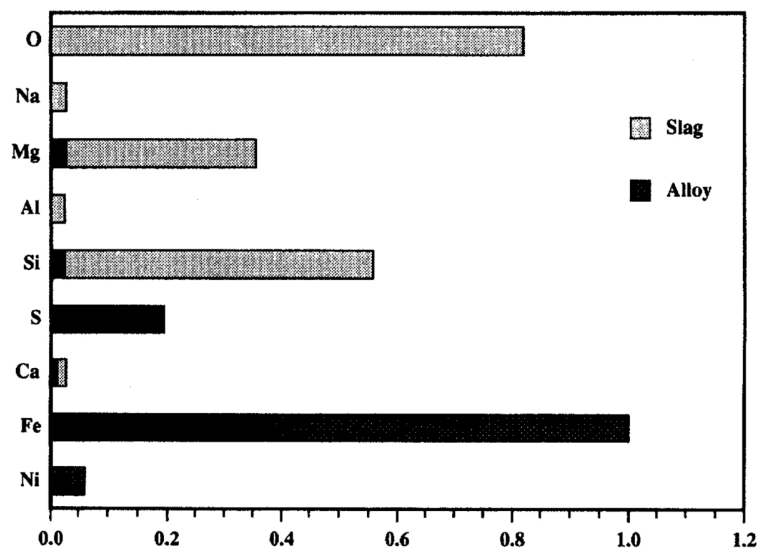

$\mathbf{E}(\mathbf{i}) / \mathbf{E}(\mathrm{Fe})$ Mass Ratio, $\mathrm{Fe}=1$

Fig. 4. Mass ratios of elements, $\mathrm{E}_{i} / \mathrm{Fe}$, as partitioned between the alloy and slag, normalized to iron $(\mathrm{Fe}=1)$. These eleven elements comprise ninety-eight percent of the mass of the Abee enstatite chondrite and by inference on the basis of Fig. 3, ninety-eight percent of the mass of the lower mantle and core of the Earth. Calculated on the basis of data from DZIEWONSKI and ANDERSON (1981), KEIL (1968), and MASON (1979). 
Table 1. Estimate of the elemental compositions of the lower mantle and total core (fluid core plus inner core) of the Earth based upon the relationship shown in Fig. 3 and the data shown in Fig. 4, calculated from DzIEwONSKI and ANDERSON (1981), KEIL (1968), and MASON (1979).

\begin{tabular}{lcc}
\hline \multicolumn{1}{c}{ Element } & Total core (grams) & Lower mantle (grams) \\
\hline Oxygen & 0 & $1.37 \times 10^{27}$ \\
Sodium & 0 & $4.60 \times 10^{25}$ \\
Magnesium & $3.89 \times 10^{25}$ & $5.49 \times 10^{26}$ \\
Aluminum & 0 & $4.22 \times 10^{25}$ \\
Silicon & $3.76 \times 10^{25}$ & $8.91 \times 10^{26}$ \\
Sulfur & $2.85 \times 10^{26}$ & 0 \\
Calcium & $1.78 \times 10^{25}$ & $2.61 \times 10^{25}$ \\
Iron & $1.46 \times 10^{27}$ & 0 \\
Nickel & $8.71 \times 10^{25}$ & 0 \\
\hline
\end{tabular}

referred to in the present paper as an alloy.

For more than a century, ideas concerning the identification of the interior portions of the Earth and other planets have been evoked by observations of meteorites, particularly iron meteorites. For example, MEUNIER (1871) envisioned meteorites as debris of a disrupted planet that had at its center a core of meteoritic iron surrounded by stone shells. The redox state of most iron meteorites (recognized by the elemental composition of the metal and by compositions of silicate inclusions) is considerably more oxidized than the redox state of the components of the Abee enstatite chondrite which are the most highly reduced naturally occurring substances known.

The chemical behaviors of elements comprising the Abee meteorite are dominated by the limited quantity of available oxygen; elements such as silicon, calcium, and magnesium would, were sufficient oxygen available, exist entirely as silicate-oxides in the slag. In most circumstances of geochemical interest, elements such as silicon, calcium, and magnesium are lithophile, given the availability of sufficient oxygen, as is the case for their occurrences in most meteorites and in rocks near the surface of the Earth. In the highly reduced matter of enstatite meteorites, on the other hand, insufficient oxygen occurs; those normally lithophile elements which are thus unable to form oxides compete on the basis of chemical activity and may be accommodated otherwise, for example as sulfides.

Approximately ninety-five percent of the mass of the Abee meteorite is comprised only of iron, magnesium, silicon, and associated oxygen and sulfur. These five elements, by virtue of their great relative abundances, constitute a buffer assemblage that effectively fixes the redox state of minor and trace elements.

The chemistry that is manifest in the alloy of the Abee meteorite and in the Earth's core, by inference on the basis of Fig. 3, may be more familiar to the steelmaker than to the geochemist. For example, in steelmaking calcium is intentionally introduced into the alloy for the purpose of precipitating calcium sulfide $(\mathrm{CaS})$ at a high temperature to desulfurate steel so that at a lower temperature iron sulfide will be unable to form (FOSTER et al., 1974). Calcium sulfide is a constituent of the non-oxide, or alloy, component of enstatite meteorites as defined in the present paper. In one instance, meteoritic calcium sulfide was observed to contain occluded grains of titanium nitride (TiN), another high-temperature metallurgical precipitate (BANNISTER, 1941). Within the Earth's core, one may expect calcium sulfide to be a high-temperature precipitate, collecting at the core-lower mantle boundary.

The slag component of the Abee meteorite consists mainly of enstatite, $\mathrm{MgSiO}_{3}(\mathrm{KEIL}, 1968)$. That $\mathrm{MgSiO}_{3}$ component, by the mass ratio relationships shown in Fig. 3, corresponds to the lower mantle of the Earth. Notably, $\mathrm{MgSiO}_{3}$, with a perovskite crystal structure, is known from experiment (LIU, 1976; ITO and MATSUI, 1978) to be stable in the pressure ranges that exist in the lower mantle of the Earth. 
Table 2. Estimate of the ${ }^{235} \mathrm{U}$ and ${ }^{238} \mathrm{U}$ content of the total core (fluid core plus inner core) of the Earth, assuming no nuclear transmutation except by natural $\alpha$ decay. Based on the Abee meteorite data of MURRELL and BURNETT (1982). Upper limits on possible lower mantle values are estimated by difference from whole-rock values.

\begin{tabular}{ccc}
\hline Uranium isotope & Total core content & $\begin{array}{c}\text { Lower mantle limit } \\
\text { (upper limit only) } \\
\text { (grams) }\end{array}$ \\
\hline Present time & $5.8 \times 10^{16}$ & $1.6 \times 10^{16}$ \\
${ }^{235} \mathrm{U}$ & $8.0 \times 10^{18}$ & $2.2 \times 10^{18}$ \\
${ }^{238} \mathrm{U}$ & $1.1 \times 10^{18}$ & $3.0 \times 10^{17}$ \\
$3 \times 10^{9}$ years ago & $1.3 \times 10^{19}$ & $3.5 \times 10^{18}$ \\
${ }^{235} \mathrm{U}$ & & \\
${ }^{238} \mathrm{U}$ & $4.9 \times 10^{18}$ & $1.3 \times 10^{18}$ \\
$4.5 \times 10^{9}$ years ago & $1.6 \times 10^{19}$ & $4.4 \times 10^{18}$ \\
${ }^{235} \mathrm{U}$ & & \\
${ }^{238} \mathrm{U}$ & &
\end{tabular}

\section{Uranium and Thorium in the Core of the Earth}

In more oxidized meteorites, uranium tends to concentrate in $\mathrm{CaO}$-rich mineral assemblages (TATSUMOTO et al., 1976). In enstatite chondrites, on the other hand, which have extremely limited oxygen, uranium and thorium appear to be chalcophile (MATSUDA et al., 1972) and occur within CaS and within [Mg,Fe]S (MURRELL and BURNETT, 1982). Both CaS and [Mg,Fe]S are components of the alloy portion of the Abee enstatite chondrite as defined in the present paper. Because of the identification of the interior portions of the Earth, the lower mantle and total core, with the slag and alloy components of the Abee enstatite chondrite (Fig. 3), one has cause to anticipate the occurrence of uranium and thorium within the core of the Earth.

Estimates of the content of uranium isotopes in the Earth's core, assuming no nuclear transmutation except by natural $\alpha$ decay, are presented in Table 2 . The values in Table 2 are calculated from data on the uranium content of $\mathrm{CaS}$ and [Mg,Fe]S of the Abee meteorite (MURRELL and BURNETT, 1982) and from data on the measured proportions of those compounds (KEIL, 1968). The difference calculated between the uranium content of those sulfides and the whole-rock value is shown in Table 2 as an upper limit on the content of uranium in the lower mantle; however, because of the experimental difficulties encountered with small samples of this extremely fine-grained, complex meteorite, that difference may not be significant and may not indicate a lithophile uranium component. The data of MURRELL and BURNETT (1982) also show that a considerable proportion of thorium exists in the $\mathrm{CaS}$ of the Abee meteorite and, by inference (Fig. 3), exists in the core of the Earth; those Abee thorium data, however, are unfortunately incomplete. The possibility can not be ruled out that the majority of the thorium in the Abee meteorite exists in the alloy portion as defined in the present paper.

\section{Concentration of Uranium in the Core of the Earth}

At ambient pressure, the crystallographic (electronic) structure of minerals greatly influences their densities. At the pressures that prevail near the center of the Earth, the density of matter becomes a function almost entirely of atomic number and atomic mass (DIRAC, 1930; MARCH, 1957). The actinides have the greatest atomic numbers $(Z \geq 90)$ and the greatest atomic masses $(A \geq 232)$ of the elements and thus comprise the most dense matter within the core of the Earth.

The chemical state of uranium in the core of the Earth is not known with certainty; generally, however, at ambient pressure, uranium compounds tend to have higher melting points than iron or nickel 


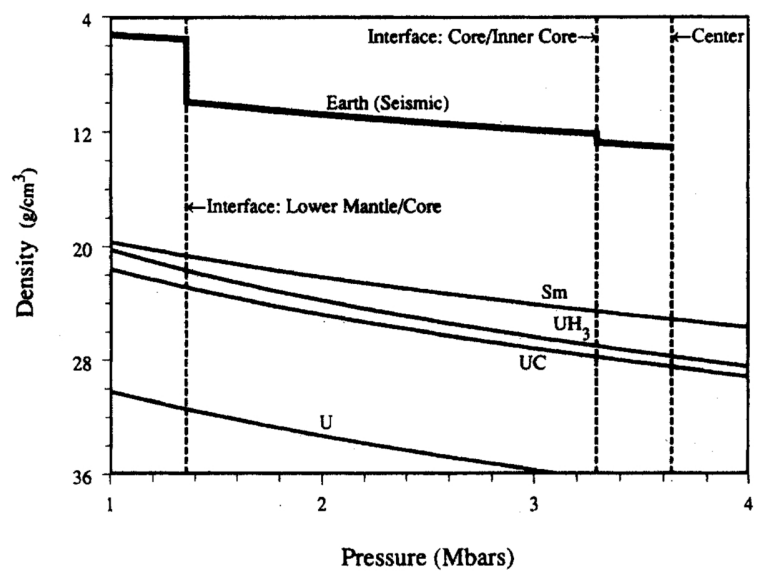

Fig. 5. Theoretical calculation of density, in $\mathrm{g} / \mathrm{cm}^{3}$, as a function of pressure, in Mbars, for uranium metal and representative compounds. The densities of thorium compounds are quite similar. The seismically determined density-pressure profile of the interior of the Earth is also shown. Sandwiched between is the density-pressure curve of samarium, representing typical fission product reactor poisons.

or their compounds (KUBASCHEWSKI and ALCOCK, 1979). Within the core of the Earth, uranium, or a compound of uranium, may therefore be expected to precipitate from the fluid core at a higher temperature than the precipitate that is the inner core (BULLEN, 1946; HERNDON, 1979) and to be the most dense solid within the core of the Earth.

Figure 5 shows density as a function of pressure for uranium metal and representative compounds, calculated using a Thomas-Fermi-Dirac approach (SALPETER and ZAPOLSKY, 1967). The compounds, uranium hydride and uranium carbide, shown in Fig. 5, were selected for presentation because these represent the least dense uranium compounds. Uranium sulfide, for example, would have a density intermediate between these and uranium metal. The densities of the actinides are considerably greater than the density of the inner core of the Earth, estimated from seismological data (DZIEWONSKI and ANDERSON, 1981). One therefore has cause to expect the action of gravity to concentrate precipitated uranium and thorium at the center of the Earth and, in doing so, to separate actinides from less dense elements which may have high neutron capture cross sections, as shown in Fig. 5 by the example of samarium.

Energy from the radioactive decay of actinides in the core of the Earth has been suggested as the energy source for the geomagnetic field (ELSASSER, 1950; FERBER et al., 1984); the possibility of a concentration of uranium at the center of the Earth undergoing self-sustaining nuclear fission chain reactions has not heretofore been suggested.

\section{Nuclear Reactor Feasibility}

In 1956, P. K. Kuroda calculated the feasibility that a terrestrial uranium deposit in the geological past might undergo self-sustaining, neutron-induced, nuclear fission reactions (KURODA, 1956). In 1972, evidence of a naturally occurring nuclear reactor was discovered at Oklo in the Republic of Gabon, Africa (BODU et al., 1972). The uranium deposit at Oklo had in fact functioned in the geological past as a natural, nuclear fission reactor. The Oklo reactor began operating about 2000 million years ago, under conditions very close to those described by KURODA (1956), and appears to have operated intermittently for a period as long as 0.6 to 1.5 million years. Besides consuming naturally occurring ${ }^{235} \mathrm{U}$ as fuel, the Oklo reactor was a breeder, producing additional ${ }^{235} \mathrm{U}$ by neutron capture in ${ }^{238} \mathrm{U}$ and subsequent decay through ${ }^{239} \mathrm{Pu}$. Furthermore, nuclear fission in the Oklo reactor occurred not only by the slow (thermal) neutron fission of ${ }^{235} \mathrm{U}$ but also to a lesser extent by the fast neutron fission of ${ }^{238} \mathrm{U}$ and ${ }^{239} \mathrm{Pu}$ (HAGEMANN et al., 1975; 
FrÉJACQUeS et al., 1975; MAURETTE, 1976; KURODA, 1982, 1983).

The concept of planetary nuclear reactors has been disclosed by the author. Jupiter, Saturn, and Neptune contain powerful internal energy sources that cause those planets to radiate approximately twice as much energy as they absorb from the Sun (PEARL et al., 1990). Observed from space, the planet Jupiter displays lateral bands of atmospheric turbulence and a more or less persistent turbulent feature, the Great Red Spot. Jupiter's atmospheric turbulence is produced by its powerful internal energy source. Recently, nuclear fission breeder reactors were suggested as internal energy sources for Jupiter, Saturn, and Neptune (HERNDON, 1992a). In the same paper, means were disclosed for concentrating the actinide elements within planetary interiors and for removing reactor poisons.

Certain of the principles that underlie the concept of planetary nuclear fission reactors, as applied to the giant gaseous planets, apply also to the core of the Earth and form the basis of the suggestion made in the present paper that the Earth contains at its center a naturally occurring nuclear fission breeder reactor as the energy source of the geomagnetic field. The present paper extends the earlier idea of hydrogeneous, thermal neutron planetary nuclear reactors, introducing the concept of fast neutron, non-hydrogeneous, planetary reactors.

Fast neutron breeder reactors consist of assemblages of fissionable nuclides for fuel and fertile nuclides for breeding; thermal neutron reactors additionally contain a light element moderator for slowing neutrons to thermal energies. The fundamental criterion for maintaining a nuclear chain reaction is that, on the average, at least one neutron produced in a fission event causes another fission event to occur. This criterion, referred to as criticality or critical condition, is described for a theoretically infinite system (FERMI, 1947) by the infinite multiplication factor, $k_{\infty}$. For a reactor to sustain a nuclear chain reaction, $k_{\infty}$ must equal or exceed 1. Calculation of the infinite multiplication factor was used in predicting the occurrence of Oklo-type nuclear reactors in uranium deposits (KURODA, 1956) and in predicting the occurrence of nuclear reactors in the giant gaseous planets (HERNDON, 1992a). At Oklo, seams of uranium ore approximately one half meter in thickness constituted a more or less "theoretically infinite" system (MAURETTE, 1976). Methods for calculating $k_{\infty}$ are described in numerous textbooks (FOSTER and WRIGHT, 1973; LAMARSH, 1983).

Individual nuclear species, such as ${ }^{235} \mathrm{U}$ and ${ }^{238} \mathrm{U}$, have unique and vastly different nuclear properties

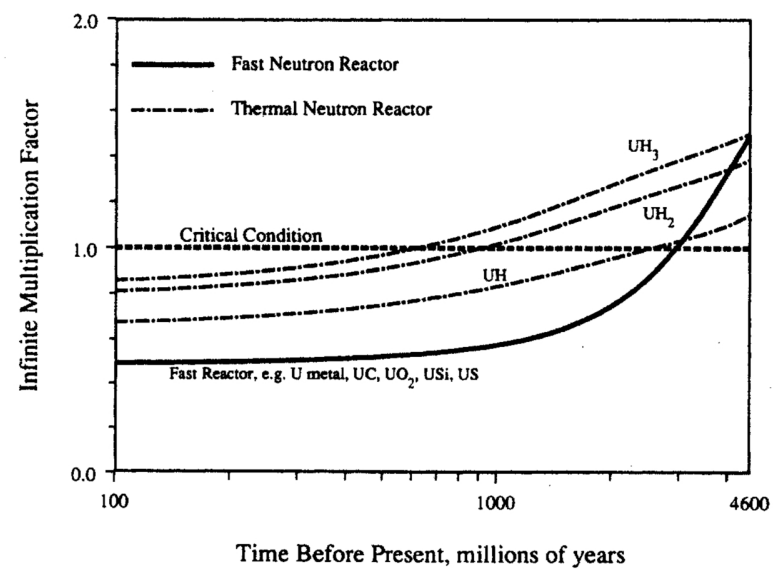

Fig. 6. The infinite multiplication factor, $k_{\infty}$, for natural uranium reactors in the geological past. In a "theoretically infinite" system, a nuclear chain reaction is possible when $k_{\infty}$ is at least equal to or exceeds unity. Because the ratio of fission cross section to capture cross section is significantly greater at high neutron energies, the fast reactor $k_{\infty}$ curve shown is approximately the same for various uranium compounds, including $\mathrm{U}$ metal, UC, $\mathrm{UO}_{2}, \mathrm{USi}$, US, and possibly others. The thermal reactor $k_{\infty}$ curves are applicable to uranium hydride compounds. This figure shows that an accumulation of uranium, or of almost any chemical compound of uranium, can in the geological past have functioned as a natural nuclear reactor. 
which enter into $k_{\infty}$ calculations, e.g. neutron absorption cross sections. Consequently, for a given system the relative proportions of the individual nuclear species dominate $k_{\infty}$ calculations. At the present time, the natural abundance of ${ }^{235} \mathrm{U}$ is too low relative to ${ }^{238} \mathrm{U}$ for naturally occurring concentrations of uranium to begin functioning as nuclear reactors and to begin to sustain a chain reaction. Because ${ }^{235} U$ has a shorter half-life than ${ }^{238} \mathrm{U}$, the abundance of ${ }^{235} \mathrm{U}$ in the past was greater relative to ${ }^{238} \mathrm{U}$. Figure 6 presents the results of $k_{\infty}$ calculations for a fast neutron reactor fueled by ${ }^{235} \mathrm{U}$ and ${ }^{238} \mathrm{U}$. The $k_{\infty}$ results are presented as a function of the ${ }^{235} \mathrm{U} /{ }^{238} \mathrm{U}$ terrestrial abundance ratio at times in the geological past. The $k_{\infty}$ calculations shown in Fig. 6 are independent of the absolute quantity of uranium in the Earth's core; the existence of a "theoretically infinite" critical mass of at least a few kilograms of uranium is assumed.

Approximately 3000 million years ago and earlier, the abundances of ${ }^{235} U$ and ${ }^{238} \mathrm{U}$ were higher (Table 2) and the ${ }^{235} U /{ }^{238} U$ terrestrial abundance ratio was sufficiently great for a "theoretically infinite" uranium assemblage to function as a fast neutron reactor, as shown in Fig. 6 by the values of $k_{\infty}$ being greater than one. Because the ratio of fission cross section to capture cross section is significantly greater at high neutron energies, the fast reactor $k_{\infty}$ curve shown in Fig. 6 is approximately the same for various uranium compounds, including U metal, UC, $\mathrm{UO}_{2}$, USi, US, and possibly others. Even though the chemical states of uranium and thorium at the center of the Earth are unknown, one may note that uranium metal or virtually any compound of uranium can, if concentrated, function as fuel for a fast neutron reactor.

The $k_{\infty}$ representation in Fig. 6, which limits fast neutron reactors to operation approximately 3000 million years ago and before, relates only to the conditions required for initial reactor operation. Subsequent continued operation depends upon fuel produced so that Fermi's $k_{\infty}$ has a value of unity or greater. An important example is the following fuel production reaction:

$$
{ }^{238} \mathrm{U}(n, \gamma)^{239} \mathrm{U} \underset{23.5 \mathrm{~m}}{\stackrel{\beta^{-}}{\longrightarrow}}{ }^{239} \mathrm{~Np} \underset{2.35 \mathrm{~d}}{\stackrel{\beta^{-}}{\longrightarrow}}{ }^{239} \mathrm{Pu} \underset{2.4 \times 10^{4} \mathrm{a}}{\stackrel{\alpha}{\longrightarrow}}{ }^{235} \mathrm{U}
$$

In calculating the fast neutron $k_{\infty}$ curve, shown in Fig. 6 , the assumption was made for each point along the curve that no prior nuclear fission had occurred. Under that assumption, approximately 3000 million years ago and later the natural $\alpha$ decay of uranium would cause the $k_{\infty}$ value to fall below unity.

A detailed treatment of nuclear reactor dynamics is extremely complicated and requires data and operating history that is unavailable. For example, other fuel breeding reactions may take place such as the following:

$$
{ }^{232} \operatorname{Th}(n, \gamma)^{233} \mathrm{Th} \underset{22.2 \mathrm{~m}}{\stackrel{\beta^{-}}{\longrightarrow}}{ }^{233} \mathrm{~Pa} \underset{27.4 \mathrm{~d}}{\stackrel{\beta^{-}}{\longrightarrow}}{ }^{233} \mathrm{U}
$$

Nevertheless, it is important to demonstrate the feasibility that a natural reactor can continue operation subsequent to 3000 million years ago. It is possible to demonstrate, at least in a simplified manner, that fuel breeding reactions can extend to the present. The breeder reaction series shown above whose end product is ${ }^{235} \mathrm{U}$ can create ${ }^{235} \mathrm{U} /{ }^{238} \mathrm{U}$ ratios that even to the present are sufficiently great that $k_{\infty}$ can maintain a value of at least unity. Such a calculation was made, based on the assumption that each atom of ${ }^{238} \mathrm{U}$ that fissions causes the formation of an atom of ${ }^{239} \mathrm{Pu}$ which then $\alpha$ decays to form an atom of ${ }^{235} \mathrm{U}$. To maintain fission to the present time the limiting condition that sufficient ${ }^{235} U$ formation from fission produced ${ }^{239} \mathrm{Pu}$ must take place in order to create ${ }^{235} \mathrm{U} /{ }^{238} \mathrm{U}$ ratios that yield $k_{\infty}$ values of at least unity. For the present calculations, the minimum amount of breeding necessary to maintain $k_{\infty}$ at unity was determined. In other words, the ${ }^{235} \mathrm{U}$ thus produced from ${ }^{239} \mathrm{Pu}$ effectively replaces the ${ }^{235} \mathrm{U}$ that would otherwise be lost due to $\alpha$ decay. As a consequence, $k_{\infty}$ can maintain a value of unity even to the present. The results of these calculations, presented in Tables 3 and 4, do not depend upon the absolute abundance of 
Table 3. Uranium isotope abundances in the core of the Earth resulting from natural $\alpha$ decay, without fission, compared to corresponding abundances calculated assuming sufficient breeding of ${ }^{235} \mathrm{U}$ from ${ }^{238} \mathrm{U}$ to maintain $k_{\infty}$ at unity.

\begin{tabular}{cllll}
\hline Years before present & $\begin{array}{c}{ }^{238} \mathrm{U} \text { (grams) } \\
\text { non-fission }\end{array}$ & $\begin{array}{c}{ }^{235} \mathrm{U} \text { (grams) } \\
\text { non-fission }\end{array}$ & $\begin{array}{c}{ }^{238} \mathrm{U} \text { (grams) } \\
\text { fission breeder }\end{array}$ & $\begin{array}{c}{ }^{235} \mathrm{U} \text { (grams) } \\
\text { fission breeder }\end{array}$ \\
\hline present & $8.16 \times 10^{18}$ & $5.74 \times 10^{16}$ & $5.44 \times 10^{18}$ & $4.39 \times 10^{17}$ \\
$1.0 \times 10^{9}$ & $9.53 \times 10^{18}$ & $1.54 \times 10^{17}$ & $7.58 \times 10^{18}$ & $6.12 \times 10^{17}$ \\
$2.0 \times 10^{9}$ & $1.11 \times 10^{19}$ & $4.11 \times 10^{17}$ & $1.01 \times 10^{19}$ & $8.14 \times 10^{17}$ \\
$3.0 \times 10^{9}$ & $1.30 \times 10^{19}$ & $1.10 \times 10^{18}$ & $1.30 \times 10^{19}$ & $1.05 \times 10^{18}$ \\
\hline
\end{tabular}

Table 4. Comparison of energy production rates for uranium in the core of the Earth for the non-fission and fission breeder cases presented in Table 4 and discussed in the text. In the non-fission case, only energy production from natural $\alpha$ decay is tabulated; in the fission breeder example, energy production is from both natural $\alpha$ decay and from sufficient fission to maintain $k_{\infty}$ at unity.

\begin{tabular}{ccc}
\hline Years before present & $\begin{array}{c}\text { Energy production } \\
\text { (ergs/sec) } \\
\text { non-fission case }\end{array}$ & $\begin{array}{c}\text { Energy production } \\
\text { (ergs/sec) } \\
\text { fission breeder case }\end{array}$ \\
\hline present & $8.0 \times 10^{18}$ & $1.1 \times 10^{22}$ \\
$1 \times 10^{9}$ & $9.8 \times 10^{18}$ & $7.2 \times 10^{21}$ \\
$2 \times 10^{9}$ & $1.3 \times 10^{19}$ & $3.1 \times 10^{21}$ \\
$3 \times 10^{9}$ & $1.9 \times 10^{19}$ & $2.7 \times 10^{20}$ \\
\hline
\end{tabular}

uranium in the core of the Earth, but upon the relative abundances of ${ }^{235} U$ and ${ }^{238} U$; the results shown, however, are calculated using the in-core uranium estimate from Table 2 and thus also provide a basis for comparing energy production rates, shown in Table 4.

The feasibility has been shown above that an accumulation uranium, or virtually any chemical compound of uranium, could have been able 3000 million years ago, to initiate self-sustaining nuclear fission reactions. Moreover, the feasibility has been demonstrated that the self-sustaining nuclear fission reactions may continue to the present through fuel breeding reactions.

Figures 1-4 and the related discussion give cause to expect substantial quantities of uranium to exist in the Earth's core. Figure 5 and the related discussion give cause to expect uranium in the core to concentrate at the center of the Earth by the action of gravity because density at core pressures is a function of atomic number and atomic mass. As shown in Fig. 6, such an accumulation of uranium, or a compound of uranium, could have been able 3000 million years ago to begin to function as a nuclear reactor. It should be emphasized that there is no known mechanism to concentrate uranium in the Earth's mantle; but, if uranium somehow had become concentrated in the mantle, in the absence of nuclei having high neutron capture cross sections, that accumulation of uranium might have begun to function as a nuclear reactor about 3000 million years ago as at least one surface concentration of uranium actually did at Oklo.

The above described calculations refer to fast neutron nuclear fission. Generally, elements of low atomic mass, which act as moderators by degrading neutron energies through scattering collisions, are not expected to concentrate at the center of the Earth because density at such a pressure is a function of atomic number and atomic mass. Even when chemically combined with uranium, light elements, such as carbon, are not sufficiently abundant to act as moderators; the exception is hydrogen.

In the interior regions of the planet Jupiter, uranium and thorium may occur as a hydrides (HERNDON, 1992a). For half a century, various ideas have been discussed suggesting the possibility of hydrides in the core of the Earth (KUHN and RITTMANN, 1941; FUKAI, 1984). Because hydrogen is an efficient moderator, 
Table 5. Examples of the components of the infinite multiplication factor used in calculations related to thermal neutron fission of $\mathrm{UH}_{3}$. From reactor theory (FERMI, 1947), $k_{\infty}=\varepsilon p f \eta$ where $\varepsilon$ is the fast fission factor, $p$ is the resonance escape probability, $f$ is the thermal utilization factor, and $\eta$ is the average number of neutrons liberated for each neutron absorbed. For the calculations, $\varepsilon=1$ was assumed.

\begin{tabular}{cccccc}
\hline $\begin{array}{c}\text { Years before } \\
\text { present }\end{array}$ & Ratio & $\begin{array}{c}\text { Resonance escape } \\
\text { probability } \\
p\end{array}$ & $\begin{array}{c}\text { Thermal } \\
\text { utilization factor } \\
{ }^{235} \mathrm{U} /{ }^{238} \mathrm{U}\end{array}$ & $\begin{array}{c}\text { Neutron } \\
\text { liberation ratio } \\
\eta\end{array}$ & $\begin{array}{c}\text { Infinite } \\
\text { multiplication factor } \\
k_{\infty}\end{array}$ \\
\hline $1.0 \times 10^{9}$ & $1.7 \times 10^{-2}$ & 0.70 & 0.93 & 1.67 & 1.09 \\
$2.0 \times 10^{9}$ & $3.8 \times 10^{-2}$ & 0.71 & 0.96 & 1.87 & 1.28 \\
$3.0 \times 10^{9}$ & $8.7 \times 10^{-2}$ & 0.71 & 0.98 & 1.98 & 1.38 \\
$4.0 \times 10^{9}$ & $2.0 \times 10^{-1}$ & 0.73 & 0.99 & 2.03 & 1.46 \\
\hline
\end{tabular}

uranium hydride can function as both fuel and moderator for a thermal neutron reactor. For generality, $k_{\infty}$ calculations were made for a thermal neutron nuclear reactor consisting of uranium hydride. The results of these calculations, also shown in Fig. 6, demonstrate the feasibility of uranium hydride compounds functioning as a thermal neutron reactor in the geological past at times when the ${ }^{235} \mathrm{U} /{ }^{238} \mathrm{U}$ terrestrial abundance ratio was greater. Data from the natural reactor at Oklo indicate that even in the presence of hydrogen some fast neutron fission and breeding occurs. As in the fast reactor case, discussed above, subsequent continued operation depends upon the fuel produced through breeding reactions. Selected values of component parameters of $k_{\infty}$ are presented in Table 5.

\section{Removal of Fission Product Poisons}

Nuclear fission reactors produce a variety of fission products. Some fission products, such as ${ }^{149} \mathrm{Sm}$, have high neutron capture cross sections and are thus referred to as reactor poisons because accumulations of these can adversely affect reactor operation. Such reactor poisons might be removed from the reactor zone at the center of the Earth by a mechanism similar in certain respects to the mechanism suggested for the accumulation of actinide elements within the Earth's core based on density differences (HERNDON, 1992a). Near the center of the Earth, the pressures are sufficiently great that the density of matter becomes a function almost entirely of atomic mass and atomic number. Reactor poisons, being fission products, have atomic masses and atomic numbers that are roughly half those of their parent actinides. The poisons therefore would have considerably lower densities than the reactor fuel and would tend to diffuse out of the region of greater density and would tend to layer on the basis of density, as shown in Fig. 5 for the example of ${ }^{149} \mathrm{Sm}$. The densities shown in Fig. 5 suggest a concentric shell of reactor poisons layered between the inner portion of the concentric shell of the inner core and the nuclear reactor sub-core at the center of the Earth. Absence of diffusion coefficient data at the relevant pressures and temperatures limits theoretical diffusion calculations. One may anticipate, however, that radiation damage might favor diffusion of the lower density reactor poisons from the region of greater density in the nuclear reactor subcore (DIENES and DAMASK, 1958). Diffusion of reactor poisons on the basis of relative density may tend to concentrate the fission products in a layer between the actinide sub-core and the central portion of the inner core without actual penetration of the inner core.

\section{Nuclear Fission Energy for Generating the Geomagnetic Field}

The geomagnetic field has been in existence at least 3000 million years (see, for example, KHRAMOV, 1982). At such a time in the past, the ${ }^{235} U /{ }^{238} \mathrm{U}$ ratio was sufficiently great that a concentration of uranium and thorium at the center of the Earth, as shown in Fig. 6, could become supercritical and function as a 
nuclear fission breeder reactor. Energy production by nuclear fission is potentially orders of magnitude greater than energy production by radioactive decay. Table 4 presents a comparison of in-core energy production rates between the above discussed non-fission example, involving only the natural $\alpha$ decay of uranium, and the fission breeder example that includes $\alpha$ decay and sufficient fission to maintain a value of unity for $k_{\infty}$. The requisite energy production rate to sustain the geomagnetic field is not known with certainty and estimates range from $10^{17}-10^{20} \mathrm{ergs} / \mathrm{sec}$; moreover, the upper limit may be biased by being constrained to limitations on energy production by previously conceived of power sources, mainly natural radioactive decay and gravitational potential energy release (JACOBS, 1975; GUBBINS and MASTERS, 1979).

I suggest that energy to sustain the geomagnetic field is produced primarily by actinide elements in the core of the Earth and by a nuclear fission breeder reactor at the center of the Earth. One might envision the inner core serving as a heat sink, a high capacity thermal reservoir, through which heat is transferred to the base of the fluid shell, creating a temperature gradient, and thus thermal convection in the electrically conducting fluid core that generates, in the manner of a self-excited dynamo, the geomagnetic field.

\section{Interrupted Reactor Operation as a Cause of Geomagnetic Field Reversals}

Approximately one half of the rocks of the Earth from all ages are magnetized in an opposite direction to the present direction of the geomagnetic field. Palaeomagnetic investigations (augmented by geological, palaeobiological, and geochronological studies) and magnetometer measurements of the ocean floor have established that the Earth's magnetic field reverses polarity frequently, but quite irregularly, with an average time between reversals of about 200,000 years. Energy sources heretofore envisioned for generating the geomagnetic field are unable to account, in a logical, causally related manner, for the recorded frequent reversals of the direction of the Earth's magnetic field over geological time. The energy sources that have been suggested change gradually and in only one direction through time (GUBBINS and MASTERS, 1979). The cause of geomagnetic field reversals has not yet been established.

Having suggested above that energy to sustain the geomagnetic field is produced by actinide elements and a nuclear fission breeder reactor at the center of the Earth, it is important to emphasize that the output energy of a naturally occurring nuclear reactor need not be constant. Various factors can alter the power output of a nuclear reactor, for example, changes in the fuel composition, physical changes in the positioning or dimensions of fuel, changes in pressure or temperature, accumulations of reactor poisons in the fuel, and possibly other effects. It is well known in nuclear reactor technology that such factors can shut down a nuclear reactor or can cause a nuclear reactor to run wild.

Nuclear fission reactors produce fission fragments, some of which, for example ${ }^{149} \mathrm{Sm}$, have high neutron capture cross sections. Fission product reactor poisons, as discussed above, may be removed from the reactor sub-core region by diffusion and gravitational layering within the inner core based on density at the prevailing pressures. One might imagine instances in which the rate of production of reactor poisons exceeds the rate of their removal. In such instances, the power output of the reactor would decrease and the reactor might shut down. When the inner core heat sink had cooled sufficiently, thermal convection in the fluid core is expected to diminish and (virtually) cease. After a period of time had elapsed for the reactor poisons to diffuse to regions of lower density, the reactor output would increase, the inner core would heat, convection would begin in the fluid core, and the Earth's magnetic field would re-establish itself, either in the same direction or in the reverse direction.

Alternatively, one might imagine circumstances within the Earth's core that could lead to cataclysmic instability within the core. For example, excess reactor heat might cause pressure to build within the core until sufficient to cause disruption in the convective motions in the fluid core and consequently decay of the Earth's magnetic field. After re-establishing quasi-static reactor operation, convection would again stabilize in the fluid core, and the magnetic field would re-establish itself with the polarity either as before or reversed. 


\section{Geophysical Implications}

Evidence has been presented in the present paper indicating that the components of the inner eightytwo percent of the Earth are virtually identical to the components of a particular meteorite, the Abee enstatite chondrite. The upper eighteen percent of the Earth appears from seismological data (DZIEWONSKI and ANDERSON, 1981) to have a complex structure characterized by numerous discontinuities and suggestive of layers of veneer. On the basis of the relationship depicted in Fig. 3, the inference is drawn that the redox state in the interior eighty-two percent of the Earth is virtually identical to the redox state of the Abee enstatite chondrite which is fixed by the buffer assemblage consisting of iron, magnesium, silicon, oxygen, and sulfur. Uranium and thorium in the inner eighty-two percent of the Earth may therefore be expected have the same redox state as in the Abee meteorite and to exist to a major extent as non-oxides in the core of the Earth.

The greatest portion of the Earth's core is fluid and contains a precipitate, the inner core. Uranium also forms precipitates that, at least at ambient pressure, have higher melting temperatures than the precipitate that is the inner core and which would, at core pressures, be more dense than any other matter within the Earth. One therefore has cause to anticipate the formation of a concentration of uranium (and thorium) as metal or as a compound at the center of the Earth. In contrast, uranium and thorium, if present in the lower mantle, would be expected to exist as lithophile large ions in the silicate phases and may not concentrate by the same mechanism as in the core.

The presence of large quantities of radioactive uranium and thorium in the core of the Earth, inferred from Fig. 3, has important implications with respect to geodynamics, particularly as manifest at the surface of the Earth, as well as with respect to geomagnetic field generation. As shown in Table 4, the rate of energy production by natural $\alpha$ decay is substantial; possible additional energy production from nuclear fission may be considerable. The fate of possible excess energy output should be considered.

Calculations based on the diffusion of heat show that heat from the core will not diffuse to the surface on a reasonable time scale; mantle silicates are good thermal insulators. Many geophysicists assume that convection occurs within the Earth's mantle and that convection currents in the mantle provide the means for moving continental plates (PELTIER, 1989), Convection, being an efficient heat transfer mechanism (SCHUBERT et al., 1979), is one way that excess energy might be removed from the core, providing, of course, that convection actually takes place in the Earth's mantle. Convection, from laboratory and industrial observations, inevitably leads to the establishment of a well defined convection cell pattern; the author has discerned no such recognizable pattern on the ocean floor. Moreover, seismic discontinuities in the upper mantle (DZIEWONSKI and ANDERSON, 1981) and chemical disequilibrium between the almost $\mathrm{FeO}$-free $\mathrm{MgSiO}_{3}$ lower mantle (Fig. 3) and $\mathrm{FeO}$-bearing rocks that are thought to be derived from within the upper mantle (JAGOUTZ et al., 1979) argue against the concept of whole-mantle convection. One may therefore wish to consider an entirely different mechanism for removing excess energy from the core that is related to the possible expansion of the Earth.

Otto HILGENBERG (1933) and, later, others observed that the continents fit together like pieces of a jigsaw puzzle on a sphere having a radius approximately half the present radius of the Earth. Hilgenberg's concept is not widely accepted, however, because scientists have heretofore lacked knowledge of a mechanism that could provide the required energy without departing from physical laws as presently known (SCHEIDEGGER, 1982). Comparing Jupiter's composition and calculated internal density with those for protoplanetary Earth, the author recently suggested the concept that the Earth is expanding from a radius of approximately $4077 \mathrm{~km}$ as it rebounds toward a new hydrostatic equilibrium after being stripped of the overburden of approximately 243 times its own mass in volatile components of a Jupiterlike protoplanet, presumably by the high temperatures and/or by the violent $\mathrm{T}$ Tauri-phase solar wind activity associated with the thermonuclear ignition of the Sun (HERNDON, 1992b). Within the framework of that concept, a different way that excess core energy might be removed from the core is by augmenting 
Earth expansion and, in doing so, supplying energy that was previously lost as heat of protoplanetary compression.

The concept of the existence of a planetary nuclear fission breeder reactor at the center of the Earth is introduced in the present paper and is supported by theoretical $k_{\infty}$ calculations demonstrating its feasibility. The concept that the Earth's geomagnetic dynamo is driven by nuclear fission energy is presented for the first time in the present paper as is the concept that the frequent, but irregular, polarity reversals of the geomagnetic field have their origins in intermittent nuclear reactor output. Although considerable uncertainty exists in estimates of the abundances of the actinide elements in the core of the Earth and in details relating to the chemistry of the core, the results of the present paper indicate that, if uranium and thorium exist in the core of the Earth as elements or compounds, as the evidence presented in this paper indicates, those actinides: (1) would be the most dense matter in the Earth; (2) would tend to concentrate at the center of the Earth; (3) would tend to be separated on the basis of density from less dense reactor poisons; and (4) would, if accumulated 3000 million years ago, be able to initiate self-sustaining nuclear fission chain reactions which may continue to the present through fuel breeding reactions. The magnitude of available nuclear fission energy release throughout geological time is of major geophysical importance and is more than sufficient to power the geomagnetic field.

I thank my sons Oliver, Joshua, and Christopher Herndon for stimulating and important discussions. I very much appreciate the comments made by Drs. P. K. Kuroda, E. E. Larson, H. S. Yoder, Jr., and the referees for the Journal of Geomagnetism and Geoelectricity.

\section{REFERENCES}

BAEDECKER, P. A. and J. T. WASSON, Elemental fractionations among enstatite chondrites, Geochim. Cosmochim. Acta, 39, 735$765,1975$.

BANNISTER, F. A., Osbornite, meteoritic titanium nitride, Mineral. Mag., 26, 36-44, 1941.

Bodu, R., H. Bouzigues, N. Morin, and J. P. Pfiffelmann, Sur 1' existence anomalies isotopiques rencontrées dan l'uranium du Gabon, C. R. Acad. Sci. Paris, 275D, 1731-1736, 1972.

Bullard, E. C., The magnetic flux within the Earth, Proc. Roy. Soc. Lond., A197, 433-453, 1949.

Bullen, K. E., A hypothesis on compressibility at pressures on the order of a million atmospheres, Nature, 157, $405,1946$.

Dienes, G. J. and Damask, A. C., Radiation enhanced diffusion, J. Appl. Phys., 29, 1713-1721, 1958.

Dirac, P. A. M., Note on exchange phenomena in the Thomas atom, Proc. Cambridge Phil. Soc., 26, 376-385, 1930.

Dziewonski, A. M. and D. A. Anderson, Preliminary reference Earth model, Phys. Earth Planet. Inter., 25, $297-356,1981$.

DzIEwONSKI, A. M. and F. GilberT, Observations of normal modes from 84 recordings of the Alaskan earthquake of 1964 March 28, Geophys. J. R. astr. Soc., 27, 393-446, 1972.

ELSASSER, W. M., On the origin of the Earth's magnetic field, Phys. Rev., 55, 489-498, 1939.

Elsasser, W. M., Induction effects in terrestrial magnetism, Phys. Rev., 69, 106-116, 1946.

Elsasser, W. M., The Earth's interior and geomagnetism, Revs. Mod. Phys., 22, 1-35, 1950.

Ferber, R. C., T. C. WALlACE, and L. M. LibBy, Uranium in the Earth's core, EOS, 65, 785-786, 1984.

FERMI, E., Elementary theory of the chain-reacting pile, Science, 105, 27-32, 1947.

Foster, A. R. and R. L. Wright, Jr., Basic Nuclear Engineering, 382 pp., Allyn and Bacon, Boston, 1973.

Foster, E., H. W. Klapdar, H. Richter, H. W. Rommerswinkel, E. SPEtzler, and J. W. Oberhausen, Stahl u. Eisen, 94, 474, 1974.

Fréjacques, C., C. Blain, C. Devillers, R. Hagemann, and J.-C. Ruffenbach, in The Oklo Phenomenon, IAEA, Vienna 509, 1975.

FUKAI, Y., The iron-water reaction and the evolution of the Earth, Nature, 308, 174-175, 1984.

Gubbins, D. and T. G. MAsters, Driving mechanisms for the Earth's dynamo, Adv. Geophys., 59, 57-99, 1979.

Hagemann, R., C. Devillers, M. Lucas, T. Lecomte, and J.-C. Ruffenbach, in The Oklo Phenomenon, IAEA, Vienna 415, 1975.

Herndon, J. M., The nickel silicide inner core of the Earth, Proc. Roy. Soc. Lond., A368, 495-500, 1979.

Herndon, J. M., The chemical composition of the interior shells of the Earth, Proc. Roy. Soc. Lond., A372, 149-154, 1980.

Herndon, J. M., The object at the centre of the Earth, Naturwissenschaften, 69, 34-37, 1982.

Herndon, J. M., Nuclear fission reactors as energy sources for the giant outer planets, Naturwissenschaften, 79, 7-14, 1992a.

HERnDON, J. M., Protoplanetary compression of the Earth and subsequent rebounding toward a new hydrostatic equlibrium, 1992b (in press). 
Hilgenberg, O. C., Vom Wachsenden Erdball, O. C. Hilgenberg, Berlin, 1933.

ITO, E. and Y. MATSUI, Synthesis and crystal-chemical characterization of $\mathrm{MgSiO}_{3}$ perovskite, Earth Planet. Sci. Lett., 38, 443$450,1978$.

JaCoBs, J. A., The Earth's Core, 253 pp., Academic Press, New York, 1975.

Jagoutz, E., H. Palme, H. Baddenhausen, K. Blum, M. Cendales, G. Dreibus, B. Spettel, V. Lorenz, and H. Wänke, The abundances of major, minor and trace elements in the earth's mantle as derived from primitive ultramafic nodules, Proc. Lunar Planet. Sci. Conf., 10, 2031-2050, 1979.

JAROSEWICH, E. and R. T. DoDD, Chemical variations among L-chondrites-IV. Analyses, with petrographic notes, of 13 L-group and LL-group chondrites, Meteoritics, 20, 23-35, 1985.

Kallemeyn, G. W., A. E. Rubin, D. WANG, and J. T. WASSON, Ordinary chondrites: Bulk compositions, classification, lithophileelement fractionations, and composition-petrographic type relationships, Geochim. Cosmochim. Acta, 53, 2747-2767, 1989.

KeIL, K., On the phase composition of meteorites, J. Geophys. Res., 67, 4055-4061, 1962.

KeIL, K., Mineralogical and chemical relationships among enstatite chondrites, J. Geophys. Res., 73, 6945-6976, 1968.

Khramov, A. N., Paleomagnetology, 308 pp., Springer-Verlag, Heidelberg, 1982.

KNOPOFF, L. and G. J. F. MACDonALD, An equation of state for the core of the Earth, Geophys. J. R. astr. Soc., 3, 68-77, 1960. Kubaschewski, O. and C. B. Alcock, Metallurgical Thermochemistry, 449 pp., Pergamon, New York, 1979.

KuHN, W. and A. RitTMANN, Über den Zustand des Erdinnern und seine Entstehung aus einem homogenen Urzustand, Geol. Rdsch., 32, 215-256, 1941.

KurodA, P. K., On the nuclear physical stability of the uranium minerals, J. Chem. Phys., 25, 781-782, 1956.

Kuroda, P. K., The Origin of the Chemical Elements and the Oklo Phenomenon, 165 pp., Springer-Verlag, Heidelberg, 1982.

KURODA, P. K., The Oklo phenomenon, Naturwissenschaften, 70, 536-539, 1983.

LAMARSH, J. R., Introduction to Nuclear Engineering, 689 pp., Addison-Wesley, Reading, Mass., 1983.

LARMOR, J., Rep. Brit. Assn., 159, 1919.

LiU, L., The high-pressure phases of $\mathrm{MgSiO}_{3}$, Earth Planet. Sci. Lett., 31, 200-208, 1976.

LOPER, D. E. and P. H. ROBERTS, Compositional convection and the gravitationally powered dynamo, in Stellar and Planetary Magnetism, edited by A. M. Soward, Gordon and Breach Sci. Pub., New York, 1983.

March, N. H., The Thomas-Fermi approximation in quantum physics, Advan. Phys., 6, 1-101, 1957.

Mason, B., Geochemistry Part 1, Meteorites, Data of Geochemistry. U.S. Geological Survey Professional Paper 440-B-1, 1979.

MATSUDA, H., M. ShimA, and M. Honda, Distribution of uranium and thorium among components of some chondrites, Geochem. $J ., 6,37-42,1972$.

Maurette, M., Fossil nuclear reactors, Ann. Rev. Nuc. Sci., 26, 319-350, 1976.

MEUNIER, M. S., Structure du globe d' ou proviennent les météorites, Compt. rend., 72, 111-113, 1871.

Murrell, M. T. and D. S. BurnetT, Actinide microdistributions in the enstatite meteorites, Geochim. Cosmochim. Acta, 46, 2453$2460,1982$.

Pearl, J. C., B. J. Conrath, R. A. Hanal, and J. A. Pirraglia, The albedo, effective temperature, and energy balance of Uranus, as determined from Voyager IRIS data, Icarus, 84, 12-28, 1990.

Peltier, W. R., Mantle Convection, 326 pp., Gordon and Breach, New York, 1989.

Ramdohr, P., The Opaque Minerals in Stony Meteorites, 245 pp., Elsevier, Amsterdam, 1973.

Reed, S. J. B., Perryite in the Kota-Kota and South Oman enstatite chondrites, Mineral. Mag., 36, 850-854, 1968.

RIKITAKE, T., Electromagnetism and the Earth's Interior, 308 pp., Elsevier, Amsterdam, 1966.

SALPETER, E. E. and H. S. ZAPOLSKY, Theoretical high-pressure equations of state including correlation energy, Phys. Rev., 158, $876-886,1967$.

Scheidegger, A. E., Principles of Geodynamics, 395 pp., Springer-Verlag, Heidelberg, 1982.

SChubert, G., P. Classen, and R. E. Young, Core cooling by subsolidus mantle convection, Phys. Earth Planet. Inter., 20, 194 208, 1979.

TAtsumoto, M., D. M. Unruh, and G. A. Desborough, U-Th-Pb and Rb-Sr systematics of Allende and U-Th-Pb systematics of Orgueil, Geochim. Cosmochim. Acta, 40, 617-634, 1976.

Verhoogen, J., Energetics of the Core, National Acad. Sci., Washington, D.C., 1980.

WAI, C. M., The metal phase of Horse Creek, Mount Egerton and Norton County enstatite meteorites, Mineral. Mag., 37, 905$908,1970$.

Wasson, J. T. and C. M. WAI, Composition of the metal, schreibersite and perryite of enstatite achondrites and the origin of enstatite chondrites and achondrites, Geochim. Cosmochim. Acta, 34, 169-184, 1970.

Wirk, H. B., On regular discontinuities in the composition of meteorites, Commentationes Physico-Mathematicae, 34, 135-145, 1969.

Note added in proof: Recently, Vidale and Benz discovered an "island" of seismically fast matter at the core-mantle boundary (Nature, 361, 529, 1993) which the author suggests is oldhamite, CaS, a high-temperature precipitate from the core, as predicted in the present paper. 